\title{
Excitability and synaptic transmission in the enteric nervous system: Does diet play a role?
}

Paul P Bertrand ${ }^{1,3}$, Kate E Polglaze ${ }^{1}$, Hui Chen ${ }^{4}$, Shaun L Sandow ${ }^{5}$, Anna Walduck ${ }^{2}$, Trisha A Jenkins ${ }^{1}$, Rebecca L Bertrand, Alan E Lomax, Lu Liu ${ }^{3}$

Schools of Medical Sciences ${ }^{1}$ and Applied Sciences ${ }^{2}$, RMIT University, Melbourne, VIC 3083, Australia

Department of Pharmacology ${ }^{3}$, University of New South Wales, Sydney, NSW 2052, Australia

School of Medical and Molecular Biosciences ${ }^{4}$, University of Technology Sydney, Sydney, NSW 2007, Australia.

Faculty of Science, Health, Education and Engineering ${ }^{5}$, University of the Sunshine Coast, Maroochydore, QLD 4558, Australia.

Running title: Dietary modulation of the ENS

* Address for Correspondence:

Paul P Bertrand, PhD

Discipline of Pharmaceutical Sciences

School of Medical Sciences and Health Innovations Research Institute

RMIT University

Melbourne, VIC 3083,

AUSTRALIA

dept: +61 29385-2804 fax: +61 29385-1059

email: dr.p.bertrand@gmail.com

Key words: high fat diet, obesity, synaptic transmission, enteric neuron.

Abbreviations: purinergic receptor P2X; cholecystokinin (CCK); serotonin (5-HT). 


\begin{abstract}
Changes in diet are a challenge to the gastrointestinal tract which needs to alter its processing mechanisms to continue to process nutrients and maintain health. In particular, the enteric nervous system (ENS) needs to adapt its motor and secretory programs to deal with changes in nutrient type and load in order to optimise nutrient absorption.
\end{abstract}

The nerve circuits in the gut are complex, and the numbers and types of neurons make recordings of specific cell types difficult, time-consuming, and prone to sampling errors. Nonetheless, traditional research methods like intracellular electrophysiological approaches have provided the basis for our understanding of the ENS circuitry. In particular, animal models of intestinal inflammation have shown us that we can documented changes to neuronal excitability and synaptic transmission.

Recent studies examining diet-induced changes to ENS programming have opted to use fast imaging techniques to reveal changes in neuron function. Advances in imaging techniques using voltage- or calcium-sensitive dyes to record neuronal activity promise to overcome many limitations inherent to electrophysiological approaches (Vanden Berghe et al., 2001). Imaging techniques allow access to a wide range of ENS phenotypes and to the changes they undergo during dietary challenges. These sorts of studies have shown that dietary variation or obesity can change how the ENS processes information - in effect reprogramming the ENS. The data gathered from intracellular recordings will be compared with measurements made using imaging techniques in an effort to determine if the lessons learnt from inflammatory changes are relevant to the understanding of diet-induced reprogramming. 


\section{Introduction}

Dramatic changes in diet macronutrient composition can present many challenges to the gastrointestinal (GI) tract. The proportion of protein, sugar and fat may change due to personal decisions or in response to medical advice. The intestine must continue to process nutrients present in the changed diet, but to do so it must update its processing mechanisms (Neunlist and Schemann, 2014). For example, the epithelium of the intestine responds quickly to nutrients by up- or down-regulating transporters and enzymes. The intestine responds to a state of over-nutrition (and indeed obesity) by increasing its absorptive capacity and by down-regulating unused transporters in times of scarcity. For example, a high sugar meal can rapidly cause new glucose transporters to be brought into play, allowing more efficient uptake - and this process may be exaggerated in diabetic patients (Young et al., 2013). The same is true for the levels of proteolytic enzymes released by the pancreas. A diet high in protein, fat or carbohydrate will lead to a progressively larger number of enzymes produced (Brannon, 1990) to facilitate more efficient digestion. Indeed, the standard animal model of obesity using the high fat diet is associated with an increase in pancreatic transcripts for lipases (Birk et al., 2014), though levels return to baseline after several months. In addition to these direct effects, diet may affect the gut microbiome, which may have indirect effects on the ENS. For example, the level of non-digestible fibre or oligosaccharides in the diet can alter the relative proportions of bacteria in the gut (Halmos et al., 2014a) and alter bowel behaviour (Halmos et al., 2014b); although the clinical implications are unknown (Camilleri and Acosta, 2014). An altered gut microbiome (Arrieta et al., 2014) is believed to be an independent regulator of intestinal function and has also been associated with obesity and other diseases such as a low grade inflammation of the GI tract. This was exemplified in a study where low dose administration of antibiotics to juvenile mice resulted not only in a shift in the microbiota populations detected, but also in an increase in adiposity in the animals 
(Cho et al., 2012).

The links between diet, inflammation and ENS function are complex, but one intriguing connection is through the multiple effects of the hormone leptin $(\mathrm{Ob})$. In addition to its central effects on satiety in the hypothalamus, leptin activates submucous and myenteric neurons (Reichardt et al., 2011, Florian et al., 2013), and has inflammatory activity as an adipocytokine. Leptin has been recognised for some time as a pro-inflammatory cytokine (La Cava and Matarese, 2004) and exerts its effects on immune function via expression of various isoforms of its receptor $(\mathrm{ObR})$ expressed on a number of immune cell populations. For example, mice with diet-induced obesity exhibited decreased T-cell activation via ObR/STAT3 signalling (Papathanassoglou et al., 2006) providing the first clear evidence for a direct link between diet, leptin, and the immune response. It reasonable to speculate that this mechanism also has the potential to link change in ENS function with change in diet and/or obesity.

Changes in nutrient type and load are probably also dealt with in the ENS by adapting its motor and secretory programs (Neunlist and Schemann, 2014). For instance, the osmolality of the chyme is maintained between $300-400 \mathrm{mOsm} / \mathrm{kg}$ in the face of a large increase in solutes as digestive enzymes break down nutrients (Kalantzi et al., 2006). Secretion is under the control of submucosal secretomotor (and vasodilator) neurons which are themselves under control from other enteric neurons that link their activity with motor patterns (Furness, 2006). Similarly, the ability of the ENS to switch from a mixing program to a propulsive program will decrease the time that luminal contents are present in the small intestine (thus increasing transit speed). This will also reduce the mixing of contents with enzymes and 
reduce the surface area of epithelium to which the contents are exposed - if taken to extremes, this will cause a reduced absorption of nutrients as can happen with chronic diarrhoea.

\section{Recording from the ENS}

The nerve circuits in the ENS responsible for motor and secretory programs are complex (Furness, 2006). In order to understand how these programs operate, researchers need to understand how the excitability of an individual neuron is regulated and how synaptic transmission between functional classes of neurons changes with disease. In order to gain this type of detailed information research methods such as intracellular electrophysiological approaches have traditionally been used. While electrophysiological techniques have provided an excellent insight into ENS function in animal models of disease, unfortunately they are difficult, time-consuming and prone to sampling errors when dealing with large numbers of neurons.

These drawbacks become more significant when looking to uncover potentially more subtle diet-induced changes in ENS behaviour. For example, while acute inflammation causes clear pathophysiological changes to gut behaviour - and an increase in the excitability of enteric neurons (Linden et al., 2003, Lomax et al., 2005b, Nurgali et al., 2007) - over-eating and the subsequent development of an obese and/or a pre-diabetic state in humans is often associated with inconsistent changes in gut behaviour (Mushref and Srinivasan, 2013). Whether there are subtle changes to enteric neuronal excitability or ENS function in these patients is even less clear (see below).

This review will focus on recent advances in fast imaging techniques that, using voltage- and 
calcium-sensitive dyes, allow recordings from many neurons on a scale previously unheard of (Vanden Berghe et al., 2001). These techniques promise to overcome many of the drawbacks listed above by allowing access to a wide range of ENS phenotypes simultaneously. However, do these techniques provide the same quality of information as do the traditional electrophysiological methods? We will explore the sorts of excitability changes in enteric neurons during dietary challenges that have been uncovered using these fast imaging techniques (Neunlist and Schemann, 2014) and will compare these with electrophysiological recordings made from animal models of inflammation.

To directly record excitability changes in enteric neurons, the action potential (AP) and the fast excitatory post-synaptic potential (EPSP) are primarily of interest. In enteric neurons, the AP has a duration of $\sim 1 \mathrm{~ms}$ and an amplitude of $\sim 100 \mathrm{mV}$ while the fast EPSP is longer at $\sim 40 \mathrm{~ms}$ but with a reduced amplitude of $\sim 10 \mathrm{mV}$ ). Thus to record both, the recording technique requires both acquisition speed and dynamic range (resolution). To record the occurrence of an AP reliably, an acquisition frequency of at least $500 \mathrm{~Hz}$ is needed. The amplitude of the AP will be lost due to undersampling, but counts of the number of APs will be accurate. With an electrophysiological approach, the speed of acquisition is really only limited by the high serial resistance of the sharp electrode - in practice, rates of $>10 \mathrm{kHz}$ yield usable data. For an imaging approach, the speed of acquisition has to be balanced against the amount of light gathered. The faster the acquisition rate, the less light is gathered which means only large signals can be seen. Current imaging cameras such as the popular Redshirt CCD system allow a $1 \mathrm{kHz}$ acquisition rate with usable signal levels and full spatial resolution of $80 \times 80$ pixels. Because the AP has such a large amplitude, the relative change in fluorescence $(\Delta \mathrm{F} / \mathrm{F})$ required is only $\sim 1 \% / 100 \mathrm{mV}$. For the longer fast EPSPs, a slower acquisition rate is acceptable, perhaps $100 \mathrm{~Hz}$, but the low amplitude means that a better 
signal to noise ratio is required such as $\Delta \mathrm{F} / \mathrm{F}$ of $\sim 10 \% / 100 \mathrm{mV}$. Worse, if changes in fast EPSP amplitude are being investigated then ideally $1 \mathrm{mV}$ resolution would be ideal. Electrophysiological approaches have both the resolution and the high signal to noise ratio required to see $1 \mathrm{mV}$ changes in membrane potential reliably. With the current imaging techniques, a $5 \mathrm{mV}$ change is probably at the limit of detection.

For the remainder of this mini-review, we will cover what traditional methods have told us about cell body excitability and synaptic transmission in enteric neurons, particularly within the context of animal models of inflammation. We will then look at diet-induced changes in enteric neurophysiology revealed by studies using fast-imaging techniques and critically review whether the lessons learnt from inflammatory changes are relevant and sufficient for understanding diet-induced reprogramming.

\section{Changes in ENS excitability during inflammation}

Most work on disease-driven changes to the ENS has been done using animal models of intestinal inflammation with a view to replicating some aspects of human Inflammatory Bowel Diseases (IBD) (Lomax et al., 2005a). These are the same types of changes - though perhaps less extreme - that we might expect to find during obesity or a pre-diabetic state, each of which have been associated with a low grade inflammation in the GI tract. Thus, it is worth reviewing the kinds of changes seen in enteric neurons during a severe inflammation as seen in animal models. There is a variety of animal models of GI inflammation including chemically induced or post-infection (Strober et al., 2002, Goyal et al., 2014). However, of all the models commonly used, significant electrophysiological recordings have only been obtained from the 2,4,6-trinitrobenzene sulfonic acid (TNBS) model of colitis in guinea pigs. 


\section{Cell body excitability of enteric neurons during inflammation}

The cell body of single enteric neurons can be recorded from in vitro preparations that have much of the local circuitry intact. Sharp conventional electrophysiological techniques can be used to record from the enteric cell body at rest, during current injection, agonist ejection, and stimulation of incoming nerve fibres to elicit synaptic potentials.

The properties of the enteric neuronal soma include the resting membrane potential (RMP), input resistance $\left(\mathrm{R}_{\mathrm{in}}\right)$ at rest and the numbers of APs elicited by stepped current injection. The RMP and the $\mathrm{R}_{\text {in }}$ at rest can be compared with similar neurons across preparations or between models of disease. However, damage during impalement can contribute to a depolarised RMP and generally a lower $R_{\text {in. }}$ If the tissue is from an inflamed gut, then dissection and impalement can cause more damage than in control tissues making comparisons difficult. To help control for these issues, many studies also use another measure of excitability. When current is injected via the recording electrode, how readily the cell body fires an AP can be determined. Some neurons may fire an AP at rest (without current injection), but generally a step increase of injected current is used to allow the number and frequency of AP firing to be recorded. In order to establish a stable comparison between different neurons in control versus an inflamed state, many studies use rheobase and the number of APs at 2x rheobase as a measurement. Rheobase is the minimum current required to elicit a single AP and $2 \mathrm{x}$ rheobase is an injection of twice that current. This allows differences in excitability that complement alterations in RMP and resting $\mathrm{R}_{\text {in }}$, but that are less susceptible to the condition of the tissue. For example, most AH neurons in the TNBS model of inflammation have more APs at 2x rheobase, while other membrane measures were 
unaffected (Lomax et al., 2005b) though a subset of AH neurons also had a depolarised RMP and increased $R_{\text {in }}$ (Nurgali et al., 2007). S neurons in the TNBS model likewise showed no changes in resting properties but a subset of orally projecting neurons had an increased number of APs at 2x rheobase (Linden et al., 2003).

Another measure of cell body excitability examines the after-hyperpolarisation (AHP) following an AP. Many neurons in the ENS have a rapid and/or sustained AHP that, when active, can severely reduce the number and frequency of APs elicited by depolarisation. Thus, the presence or absence of the AHP in the AH/sensory neurons is another measure of excitability (Linden et al., 2003) which computer modelling suggests is the key to the overall state of the circuitry (Thomas et al., 2000). In myenteric and submucosal AH neurons from TNBS treated animals, the AHP was substantially reduced (Linden et al., 2003, Lomax et al., 2005b, Nurgali et al., 2007).

\section{Fast synaptic transmission in the ENS during inflammation}

Most enteric neurons respond to electrical stimulation of interganglionic fibre tracts with the generation of a fast EPSP. Enteric neurons in the electrophysiological 'S' class have fast EPSPs by definition, while under some conditions AH neurons (those with the sustained AHP) have occasionally been seen to have a fast EPSP. In S neurons, at least three ligandgated ion channels contribute to fast EPSPs, with the nicotinic receptor sub-types accounting for the majority in myenteric (Galligan, 2002) and submucous (Monro et al., 2004) neurons. There are also P2X-mediated fast EPSPs (Galligan and Bertrand, 1994), especially between particular sub-types of enteric neurons (e.g., descending interneurons to inhibitory motor neurons (Bian et al., 2000)), and a relatively minor contribution from 5-HT3 receptors (e.g., in 
descending motor pathways (Monro et al., 2002) and secretory pathways (Monro et al., 2008)). The $\mathrm{P} 2 \mathrm{X}$ and 5- $\mathrm{HT}_{3}$ receptor subtypes involved are not clear, but there is evidence that P2X2 and P2X3 receptors are major players (Ren et al., 2003) while mRNA has been found for a variety of 5- $\mathrm{HT}_{3}$ receptor sub-types (Kapeller et al., 2011).

Although nicotinic fast synaptic transmission between enteric neurons is the most common in all species studied (including humans), the proportion of nicotinic neurotransmission can change in animal models of inflammation. In particular, Lomax et al. showed that the larger fast EPSPs in the submucosal neurons were due to an increase in non-nicotinic transmission via 5- $\mathrm{HT}_{3}$ and $\mathrm{P} 2 \mathrm{X}$ receptors (Lomax et al., 2005b). As mentioned above, non-nicotinic transmission may be more important at particular functional types of synapse, thus, inflammation induced changes may have differential effects on these pathways. Finally, while the S neurons have fast EPSPs that are altered during inflammation, Linden et al. found the myenteric AH neurons that normally do not have a fast EPSP were more likely to have a nicotinic fast EPSP in TNBS-inflamed distal colon (Linden et al., 2003).

Most if not all enteric neurons also respond to trains of electrical stimuli at interganglionic fibre tracts with a slow EPSP mediated by G-protein coupled receptors. Slow EPSPs are also associated with increases in cell body excitability and, thus, it is not surprising that in neurons from inflamed tissues, the duration of the slow EPSP is often longer lasting (Nurgali et al., 2007).

\section{Studies of enteric neurons during obesity and/or a high fat diet}

Because of the short-comings of traditional electrophysiological methods discussed 
previously, recent studies have opted to use fast imaging techniques (Vignali et al., 2010) using voltage or calcium sensors to examine diet-induced changes to ENS programming.

One of the first studies to look at changes to the ENS that might accompany alterations in diet was Reichardt et al. (2011) where the anorexigenic hormone leptin was examined. Leptin is released from adipocytes and supresses central appetite signalling and their hypothesis was that peripheral effects of leptin at the level of the ENS were present. They used a fast voltage-sensitive dye (di-8-ANEPPS acquired at $1000 \mathrm{~Hz}$ ) to show that microejection of leptin onto an enteric ganglion excited a small population of both myenteric and submucosal neurons from the guinea pig colon. The excitation consisted of a low frequency train of APs with a time course similar to that seen when nicotine was used. In the case of pressure ejected nicotine, the APs are generated by a large depolarisation mediated by the nicotinic receptor; as characterised in electrophysiological studies (e.g., Galligan and Bertrand, 1994). In the case of leptin, the constraints of high pass filtering of the imaging data do not allow a slow depolarisation to be seen directly, but the data are consistent with leptin causing a low amplitude depolarisation leading to AP firing. It is well accepted that in high-fat diet induced obesity, there is high level of blood leptin due to increased adiposity while leptin resistance is also developed subsequently which can compromise the action of leptin in the brain. Whether the same leptin resistance is developed in the obese ENS is currently unclear.

The first study to examine changes to the ENS as a result of altered diet was Baudry et al. (2011). In the antrum of mice fed a Western diet, that was a modified chow which was high in fat and simple sugars, for 12 weeks. 'Fast' calcium imaging (Fluo-4 acquired at $40 \mathrm{~Hz}$ ) recorded calcium transients and absolute levels following a train of electrical stimuli to an 
interganglionic fibre tract. The calcium transients are likely to represent a fast EPSP with an AP riding on top (Shuttleworth and Smith, 1999, Vanden Berghe et al., 2000, Michel et al., 2011), while an increase in the absolute level of intracellular calcium may represent a depolarisation of the membrane potential similar to a slow EPSP (Vanden Berghe et al., 2000). It was shown that the Western diet was not associated with a change in the number of calcium transients (i.e., APs) following nerve stimulation, but that the slow increase in baseline calcium was $>50 \%$ larger. The slow increase could relate to a larger individual fast EPSP with a greater accumulation of calcium, and/or there may be a larger slow EPSP that also contributes to the increased baseline.

Roosen et al. (2012) investigated how enteric neurons from guinea pig respond to the neurotransmitter/enteroendocrine transmitter 5-HT, the enteroendocrine transmitter cholecystokinin (CCK), and the orexigenic hormone ghrelin. Ghrelin is released from enteroendocrine cells in the stomach and intestine to increase central appetite signalling while on the other hand CCK and 5-HT are likely to cause suppression of appetite signalling. 'Fast' calcium imaging (Fluo-4, probably acquired at $40 \mathrm{~Hz}$ ) showed that enteric neurons from fasted guinea pigs were more sensitive to ghrelin while re-fed animals were more sensitive to CCK. Although not truly fast, the calcium signals have been shown to correlate well with AP firing (Martens et al., 2014). Electrical stimulation of fibre tracts caused more neurons to respond with larger responses in re-fed animals versus fasted. In contrast, ghrelin produced larger calcium transients in neurons from fasted animals. Moreover CCK caused a greater number of neurons to respond in the re-fed state, while 5-HT responses were unaltered. A solution with high potassium $(75 \mathrm{mM})$ was used to depolarise the myenteric neurons in a way similar to current injection. There was an increase in the amplitude of the calcium transient following high potassium in re-fed animals suggesting that the membrane 
potential may already have been partially depolarised, and thus more excitable, in the re-fed state.

Most recently, Reichardt et al. (2013) used mice fed a Western diet to examine changes in myenteric neuron excitability in the mouse distal colon. A fast voltage-sensitive dye (di-8ANEPPS acquired at $1000 \mathrm{~Hz}$ ) enabled exploration of changes in response to microejection of the neurotransmitters analogues nicotine and 2-methyl 5-HT that activate receptors that normally mediate enteric fast EPSPs. The main finding from this research was that 12 weeks of the Western diet increased the number of neurons responding to nicotinic and serotonergic activation from 5-10\% while the frequency of AP discharge was unaffected. The correlation between weight and magnitude of response was significant with the heaviest Western diet fed mice having the most responsive neurons to both agonists. The increased responsiveness to nicotine and 2-methyl 5-HT suggests that fast EPSPs in a population of neurons would also be larger. Unfortunately, the types of neurons activated were not revealed by immunohistochemical processing.

Together, these types of studies show that diet can change how the ENS processes information. However, the measures of excitability used by these studies are quite different from those used in the intracellular electrophysiology studies examining inflammation. Rather than using depolarising current to look at action potential discharge, imaging studies have relied mainly on exogenous application of agonists such as compounds thought to be involved in the regulation of food intake or those that are involved in fast synaptic transmission. To date, no imaging studies have looked at fast EPSP amplitude or composition during changes in diet. However, such studies are technically possible as the 
voltage sensitive dyes have the resolution to detect fast EPSP amplitude directly (Bertrand et al., 2009); such that the proportions of nicotinic versus other fast transmitters can be assessed in diet-induced obesity.

\section{Key methodological issues for fast imaging of enteric neuronal excitability}

Do these fast imaging techniques replace or complement traditional electrophysiological approaches? Several key issues and potential short-comings remain with the imaging studies such as: can individual neurons be uniquely identified by their neurochemical code (or projection pattern); if fast and slow synaptic transmission can be assessed quantitatively; whether signals can be uniquely related to single neurons, dendrites or fibres of passage; and can changes in neuronal activity equate to a difference in organ behaviour.

The functional identity of the neurons recorded from is crucial to understanding their role in the ENS circuitry. The functional class is generally determined by post-experimental immunohistochemical processing to reveal key neuronal markers. While individual neurons can be uniquely identified following intracellular recording (and injection of a marker such as neurobiotin), fast imaging studies must rely on the position of the neuron within the ganglia to match images of activity versus images of neuronal markers. To complicate matters, initial images are in fresh tissue, while latter images are of fixed tissues with sometimes distorted tissue dimensions relative to that in situ. One step forward in this area is the use of genetically encoded calcium indicators (Boesmans et al., 2013). Currently these indicators are expressed in all enteric neurons and thus give much the same data as exogenously applied indicators, but directed expression in a particular class of neuron (e.g., NOS expressing neurons) would provide much needed data on how a population of such neurons behaves 
within the ENS circuitry.

Typically, different classes of enteric neuron are dispersed within a ganglion; though there are some rules of thumb (Smith et al., 1999). In practice, this means that dissimilar neurons are often immediate neighbours. With intracellular recordings from the cell body, electrical signals are restricted to a single compartment (i.e., the neuronal soma) with changes in electrically distant parts of the neuron (such as the dendrites) contributing less to signals than those at the cell body. With imaging, all parts of the neuron can theoretically contribute to the signal, but in practice, the dendrites of one neuron may be too spatially distant, or indistinct to be able to attribute to a particular cell body. Fibres of passage also contribute to the electrical signal in voltage sensitive dye experiments, and care must be taken to restrict regions of interest to the main soma of neurons. In small ganglia with only a single layer of neurons this is less of a problem, while large myenteric ganglia with two layers of neurons and fibres can present a more significant technical issues.

Whether the changes seen in individual neurons equate to differences in whole organ behaviour has been a perennial problem when examining enteric neuron activity. Sharp electrophysiological recordings suffer greatly in this regard as only a few neurons of a particular class may be recorded from during a series of experiments. Changes seen in a few neurons may not equate into changes in a large number of neurons, and with gut behaviour most responses are likely to be due to large numbers of neurons (e.g., the migrating motor complex - MMC (Thomas et al., 2004)). Fast imaging can record from many more neurons increasing the likelihood that any changes seen in a particular class of neuron are typical, and thus may translate into a change in organ physiology. 


\section{Conclusions}

Under normal conditions, the gut is well-equipped to extract nutrients from a meal. In times of food scarcity this is a benefit, but in times of plenty this system may extract too many nutrients, contributing to obesity. In this situation, the ENS needs to learn to accommodate and potentially ignore large nutrient loads. Conversely, diseased or shortened intestine may struggle to avoid malnutrition and perhaps there is a way in which the ENS can be fine-tuned to allow greater absorption. Recent studies have shown that diet and/or obesity can change how the ENS processes information. Lessons learnt from inflammatory changes in the ENS provide a good starting point in trying to understand diet-induced reprogramming. 


\section{References}

Arrieta MC, Stiemsma LT, Amenyogbe N, Brown EM, Finlay B (2014) The intestinal microbiome in early life: health and disease. Front Immunol 5:427.

Baudry C, Reichardt F, Marchix J, Bado A, Schemann M, Bruley des Varannes S, Neunlist M, Moriez R (2011) Diet-induced obesity has neuroprotective effects in murine gastric enteric nervous system: involvement of leptin and GDNF. J Physiol (Lond) 590:533-544.

Bertrand RL, Michel K, Schemann M, Bertrand PP (2009) Exploring short term plasticity in the enteric nervous system: Voltage-sensitive dye recordings of guinea pig myenteric neurons. In: Autonomic Neuroscience: Basic and Clinical, vol. 149, p 96.

Bian X-C, Bertrand PP, Bornstein JC (2000) Descending inhibitory reflexes involve P2X receptormediated transmission from interneurons to motor neurons in guinea-pig ileum J Physiol (Lond) 528:551-560.

Birk RZ, Rubio-Aliaga I, Boekschoten MV, Danino H, Muller M, Daniel H (2014) Differential regulation of pancreatic digestive enzymes during chronic high-fat diet-induced obesity in C57BL/6J mice. Br J Nutr 112:154-161.

Boesmans W, Martens MA, Weltens N, Hao MM, Tack J, Cirillo C, Vanden Berghe P (2013) Imaging neuron-glia interactions in the enteric nervous system. Front Cell Neurosci 7:183.

Brannon PM (1990) Adaptation of the exocrine pancreas to diet. Annu Rev Nutr 10:85-105.

Camilleri M, Acosta A (2014) Re: Halmos et al, A diet low in FODMAPs reduces symptoms of irritable bowel syndrome. Gastroenterology 146:1829-1830.

Florian V, Caroline F, Francis C, Camille S, Fabielle A (2013) Leptin modulates enteric neurotransmission in the rat proximal colon: an in vitro study. Regul Pept 185:73-78.

Furness JB (2006) The Enteric Nervous System. Oxford, UK: Wiley-Blackwell.

Galligan JJ (2002) Ligand-gated ion channels in the enteric nervous system. Neurogastroenterol Motil 14:611-623.

Galligan JJ, Bertrand PP (1994) ATP mediates fast synaptic potentials in enteric neurons. J Neurosci 14:7563-7571.

Goyal N, Rana A, Ahlawat A, Bijjem KR, Kumar P (2014) Animal models of inflammatory bowel disease: a review. Inflammopharmacology 22:219-233.

Halmos EP, Christophersen CT, Bird AR, Shepherd SJ, Gibson PR, Muir JG (2014a) Diets that differ in their FODMAP content alter the colonic luminal microenvironment. Gut.

Halmos EP, Power VA, Shepherd SJ, Gibson PR, Muir JG (2014b) A diet low in FODMAPs reduces symptoms of irritable bowel syndrome. Gastroenterology 146:67-75 e65.

Kalantzi L, Goumas K, Kalioras V, Abrahamsson B, Dressman JB, Reppas C (2006) Characterization of the human upper gastrointestinal contents under conditions simulating bioavailability/bioequivalence studies. Pharm Res 23:165-176.

Kapeller J, Moller D, Lasitschka F, Autschbach F, Hovius R, Rappold G, Bruss M, Gershon MD, Niesler B (2011) Serotonin receptor diversity in the human colon: Expression of serotonin type 3 receptor subunits 5-HT3C, 5-HT3D, and 5-HT3E. J Comp Neurol 519:420-432.

La Cava A, Matarese G (2004) The weight of leptin in immunity. Nat Rev Immunol 4:371-379.

Linden DR, Sharkey KA, Mawe GM (2003) Enhanced excitability of myenteric AH neurones in the inflamed guinea-pig distal colon. J Physiol (Lond) 547:589-601.

Lomax AE, Fernandez E, Sharkey KA (2005a) Plasticity of the enteric nervous system during intestinal inflammation. Neurogastroenterol Motil 17:4-15. 
Lomax AE, Mawe GM, Sharkey KA (2005b) Synaptic facilitation and enhanced neuronal excitability in the submucosal plexus during experimental colitis in guinea-pig. J Physiol 564:863-875.

Martens MA, Boesmans W, Vanden Berghe P (2014) Calcium imaging at kHz frame rates resolves millisecond timing in neuronal circuits and varicosities. Biomed Opt Express 5:2648-2661.

Michel K, Michaelis M, Mazzuoli G, Mueller K, Vanden Berghe P, Schemann M (2011) Fast calcium and voltage-sensitive dye imaging in enteric neurones reveal calcium peaks associated with single action potential discharge. J Physiol 589:5941-5947.

Monro RL, Bertrand PP, Bornstein JC (2002) ATP and 5-HT are principal neurotransmitters in the descending excitatory reflex pathway of the guinea-pig ileum. Neurogastroenterol Motil $14: 255-264$.

Monro RL, Bertrand PP, Bornstein JC (2004) ATP participates in three excitatory post-synaptic potentials in the submucous plexus of the guinea pig ileum. J Physiol (Lond) 556:571-584.

Monro RL, Bornstein JC, Bertrand PP (2008) Synaptic transmission from the submucosal plexus to the myenteric plexus in Guinea-pig ileum. Neurogastroenterol Motil 20:1165-1173.

Mushref MA, Srinivasan S (2013) Effect of high fat-diet and obesity on gastrointestinal motility. Ann Transl Med 1:14.

Neunlist M, Schemann M (2014) Nutrient-induced changes in the phenotype and function of the enteric nervous system. J Physiol 592:2959-2965.

Nurgali K, Nguyen TV, Matsuyama H, Thacker M, Robbins HL, Furness JB (2007) Phenotypic changes of morphologically identified guinea-pig myenteric neurons following intestinal inflammation. J Physiol 583:593-609.

Papathanassoglou E, El-Haschimi K, Li XC, Matarese G, Strom T, Mantzoros C (2006) Leptin receptor expression and signaling in lymphocytes: kinetics during lymphocyte activation, role in lymphocyte survival, and response to high fat diet in mice. J Immunol 176:7745-7752.

Reichardt F, Baudry C, Gruber L, Mazzuoli G, Moriez R, Scherling C, Kollmann P, Daniel H, Kiesling S, Haller D, Neunlist M, Schemann M (2013) Properties of myenteric neurons and mucosal functions in the distal colon of diet-induced obese mice. J Physiol.

Reichardt F, Krueger D, Schemann M (2011) Leptin excites enteric neurons of guinea-pig submucous and myenteric plexus. Neurogastroenterol Motil 23:e165-170.

Ren J, Bian X, DeVries M, Schnegelsberg B, Cockayne DA, Ford AP, Galligan JJ (2003) P2X2 subunits contribute to fast synaptic excitation in myenteric neurons of the mouse small intestine. J Physiol (Lond) 552:809-821.

Roosen L, Boesmans W, Dondeyne M, Depoortere I, Tack J, Vanden Berghe P (2012) Specific hunger- and satiety-induced tuning of guinea pig enteric nerve activity. J Physiol 590:43214333.

Shuttleworth CW, Smith TK (1999) Action potential-dependent calcium transients in myenteric S neurons of the guinea-pig ileum. Neuroscience 92:751-762.

Smith TK, Burke EP, Shuttleworth CW (1999) Topographical and electrophysiological characteristics of highly excitable $\mathrm{S}$ neurones in the myenteric plexus of the guinea-pig ileum. J Physiol (Lond) 517 ( Pt 3):817-830.

Strober W, Fuss IJ, Blumberg RS (2002) The immunology of mucosal models of inflammation. Annu Rev Immunol 20:495-549.

Thomas EA, Bertrand PP, Bornstein JC (2000) A computer simulation of recurrent, excitatory networks of sensory neurons of the gut in guinea-pig. Neurosci Lett 287:137-140.

Thomas EA, Sjovall H, Bornstein JC (2004) Computational model of the migrating motor complex of the small intestine. Am J Physiol 286:G564-572. 
Vanden Berghe P, Bisschops R, Tack J (2001) Imaging of neuronal activity in the gut. Curr Opin Pharmacol 1:563-567.

Vanden Berghe P, Tack J, Coulie B, Andrioli A, Bellon E, Janssens J (2000) Synaptic transmission induces transient $\mathrm{Ca} 2+$ concentration changes in cultured myenteric neurones. Neurogastroenterol Motil 12:117-124.

Vignali S, Peter N, Ceyhan G, Demir IE, Zeller F, Senseman D, Michel K, Schemann M (2010) Recordings from human myenteric neurons using voltage-sensitive dyes. J Neurosci Methods 192:240-248.

Young RL, Chia B, Isaacs NJ, Ma J, Khoo J, Wu T, Horowitz M, Rayner CK (2013) Disordered control of intestinal sweet taste receptor expression and glucose absorption in type 2 diabetes. Diabetes. 